\title{
Circle-Representations of Simple 4-Regular Planar Graphs ${ }^{\star}$
}

\author{
Michael A. Bekos ${ }^{1}$ and Chrysanthi N. Raftopoulou ${ }^{2}$ \\ 1 University of Tübingen, Institute for Informatics, Germany \\ bekos@informatik. uni-tuebingen.de \\ 2 School of Applied Mathematical \& Physical Sciences, \\ National Technical University of Athens, Greece \\ crisraft@mail.ntua.gr
}

\begin{abstract}
Lovász conjectured that every connected 4-regular planar graph $G$ admits a realization as a system of circles, i.e., it can be drawn on the plane utilizing a set of circles, such that the vertices of $G$ correspond to the intersection and touching points of the circles and the edges of $G$ are the arc segments among pairs of intersection and touching points of the circles. In this paper, (a) we affirmatively answer Lovász's conjecture, if $G$ is 3-connected, and, (b) we demonstrate an infinite class of connected 4-regular planar graphs which are not 3 -connected and do not admit a realization as a system of circles.
\end{abstract}

\section{Introduction}

All graphs considered in this paper are simple, finite and undirected. Given a graph $G$, we denote by $V[G]$ and $E[G]$ the set of vertices and edges of $G$, respectively. If $G$ is regular, we denote by $d(G)$ its degree.

Definition 1. Let $G$ be a connected 4-regular planar graph. We say that $G$ admits a realization as a system of circles, if it can be drawn on the plane using a set of circles such that (see Figures $1 b, 1 d$ ): (1) The vertex set $V[G]$ is given by the intersection and touching points of the circles, (2) the edge set $E[G]$ is defined by all circular arcs between the intersection and touching points of the circles. In the special case where intersection points are not allowed, we say that $G$ admits a realization as a system of touching circles (see Figures 16 and [1]).

Lovász [7, pp.1175], [11, pp.426] conjectured that every simple connected 4-regular planar graph admits a realization as a system of circles. To the best of our knowledge this conjecture remained unanswered. Touching points are necessary, since if we use only crossings, we have an even number of vertices, but there are 4-regular planar graphs with odd number of vertices [14].

\footnotetext{
* The work of M.A. Bekos is implemented within the framework of the Action "Supporting Postdoctoral Researchers" of the Operational Program "Education and Lifelong Learning" (Action's Beneficiary: General Secretariat for Research and Technology), and is co-financed by the European Social Fund (ESF) and the Greek State.
} 


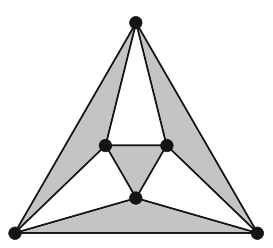

(a)

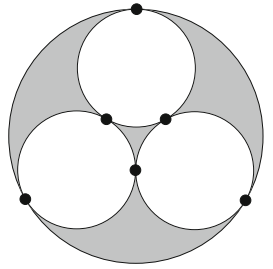

(b)

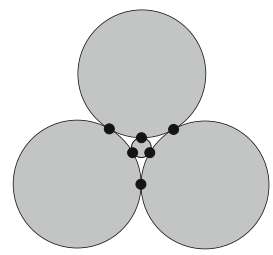

(c)

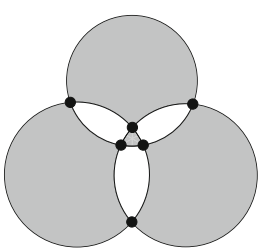

(d)

Fig. 1. (a) A straight-line drawing of the octahedron graph $G_{\text {oct }}$. (b)-(d) Different realizations of the octahedron graph $G_{\text {oct }}$ as system of circles

In this paper, we prove that using touching points of circles as vertices is indeed sufficient in order to affirmatively answer Lovász's conjecture in the case of 3-connected graphs. If the input graph is not 3-connected, we demonstrate by an example that a realization as a system of circles is not always possible.

\section{Related Work}

Closely related to the problem we study is the contact graph representation problem. A contact graph is a graph whose vertices are represented by geometric objects and its edges correspond to two objects touching in some specific predefined way. There is a rich literature on contact graphs (cf. 9 8]), dating back to 1936 in Koebe's theorem [10] which states that any planar graph can be represented as a contact graph of disks in the plane.

Lombardi drawings, which attempt to capture some of the visual aesthetics used by the American artist Mark Lombardi, are also closely related to our problem. Two features that stand out in Lombardi's work are the use of circulararc edges and their even distribution around vertices. Such even spacing of edges around each vertex (also known as perfect angular resolution) together with the usage of circular arc edges, formally define Lombardi drawings of graphs [6 5,4].

Connected 4-regular planar graphs is a well studied class of graphs. Manca [14] proposed four operations to generate all connected 4-regular planar graphs from the octahedron graph. As noted by Lehel [11, Manca's construction could not generate all connected 4-regular planar graphs, however, an additional operation could fix this problem. Broersma et. al [3] showed that all 3-connected 4-regular planar graphs can also be generated from the octahedron, using three operations.

In the context of graph drawing, 4-regular planar graphs have a long tradition, dating back to VLSI layouts and floor-planning applications. The main goal in this context is to produce drawings (referred to as orthogonal drawings) in which each vertex corresponds to a point on the integer grid and each edge is represented by a sequence of horizontal and vertical line segments. Pioneering work on orthogonal drawings was done in relation to VLSI-design by Valiant [17, Leiserson [13] and Leighton [12] and later on, in relation to graph drawing by Tamassia [15], Tamassia and Tollis [16], and Biedl and Kant [1. 


\section{The Case of 3-Connected 4-Regular Planar Graphs}

We first prove that a 3-connected 4-regular planar graph admits a realization as a system of touching circles. It is well known that a connected planar graph is Eulerian if and only if its dual is bipartite [2, pp.172]. Let $G$ be an embedded 4-regular planar graph. Since $G$ is Eulerian, its dual $G^{*}$ is bipartite. Hence, we can color the faces of $G$ using two colors, say gray and white, so that any two adjacent faces are of different colors. For convenience, we assume that the outer face of $G$ is always colored white. We proceed to construct a new graph $I L(G)$ as follows. We associate a vertex of $I L(G)$ with every gray face of $G$. We join two vertices of $I L(G)$ with an edge if and only if the corresponding faces of $G$ have at least one vertex in common (refer to the black colored graph of Fig 2a).

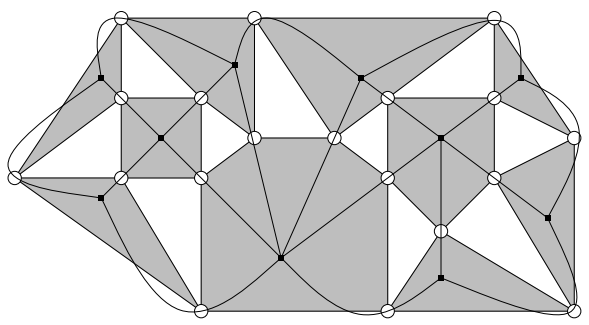

(a)

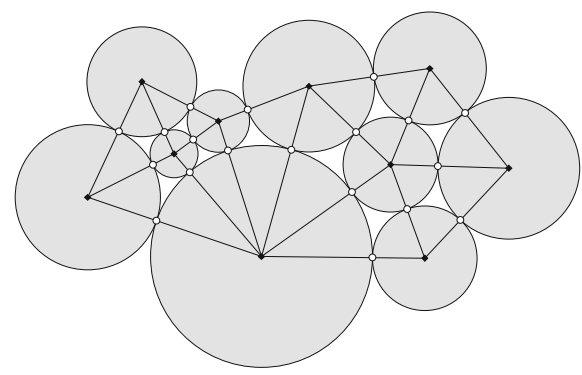

(b)

Fig. 2. (a) Constructing graph $I L(G)$. (b) A realization of $G$ as system of circles.

Lemma 1. If $G$ is simple 3-connected 4-regular planar, then $I L(G)$ is simple.

Proof. Suppose that $G$ is 3-connected and assume to the contrary that $I L(G)$ is not simple. W.l.o.g., we further assume that $I L(G)$ contains a multiple edge, say a double edge between $f$ and $g$, where $f, g \in V[I L(G)]$ (see Fig[3). The case where $I L(G)$ contains selfloops is treated similarly. By definition, $f$ and $g$ correspond to gray faces of $G$ that have exactly two common vertices, say $u, v \in V[G]$. Then, $u, f, v$ and $g$ define a separating simple closed curve which intersects $G$ at exactly two vertices. Note that since $G$ is simple there is at least one vertex of $G$ that lies in the interior of this curve and one on its exterior; a clear contradiction.

Theorem 1. Every simple 3-connected 4-regular planar graph admits a realization as a system of touching circles.

Proof. By Lemma 1, $I L(G)$ is simple. So, by applying Circle-Packing theorem [10 on it, we obtain a drawing in which each gray-colored face of $G$ corresponds to a circle and two circles meet at a point if and only if the corresponding faces are vertex-adjacent. Hence, the points where the circles meet define the vertices of $G$ and the arc segments between them correspond to the edges of $G$. 


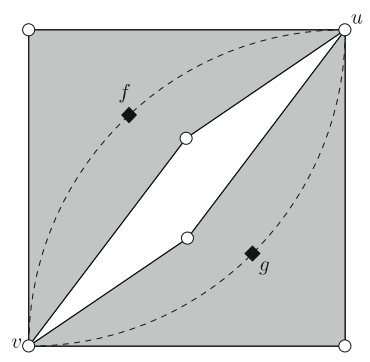

Fig. 3. Vertices $u, f, v$ and $g$ define a separating simple closed curve

\section{The General Case of Connected 4-Regular Planar Graphs}

In this section, we will demonstrate an infinite class of connected 4-regular planar graphs that do not admit a realization as a system of circles. Hence, Lovász's conjecture does not hold for every simple connected 4-regular planar graph. Note that in the case where $G$ is not 3-connected, $I L(G)$ is not necessarily simple. Hence, the Circle-Packing theorem cannot be applied directly.

The base of our constructive proof is the octahedron graph $G_{\text {oct }}$ (see Fig 1a), which is a simple 3-connected 4-regular planar graph. Hence, Theorem 1 suggests that it admits a realization as a system of touching circles. The following lemma describes all different realizations of $G_{\text {oct }}$ as a system of circles.

Lemma 2. The octahedron graph has exactly three different realizations as a system of circles, which are demonstrated in Figures $1 b]$, 1d.

Proof. In general, there are certain restrictions concerning the number of circles that participate in a realization of a graph as a system of circles. Two circles may have at most two vertices in common (two crossing points if they intersect, one touching point if they are tangent, or none if they are separated), there are at least three vertices on every circle (since we consider only simple graphs), and, every vertex belongs to exactly two circles (since every vertex has degree 4).

Translating these properties back to the octahedron graph $G_{o c t}$, we obtain the following: If every pair of circles contributes exactly two vertices, we may have a minimum of three circles, since the octahedron graph consists of six vertices. Refer to Fig $1 \mathrm{~d}$ for such a realization. On the other hand, if every circle has exactly three vertices, and therefore defines exactly three edges, we have a maximum of four circles, since the octahedron graph consists of 12 edges. Figures $1 \mathrm{~b}$ and $1 \mathrm{c}$ depict such realizations.

From the above, it follows that any realization of $G_{\text {oct }}$ as a system of circles consists of either three or four circles. This implies, that there exist exactly three different realizations of $G_{o c t}$ as a system of circles: one representation using three circles, and two representations using four circles, depending on whether the outerface is bounded by a circle or not. 


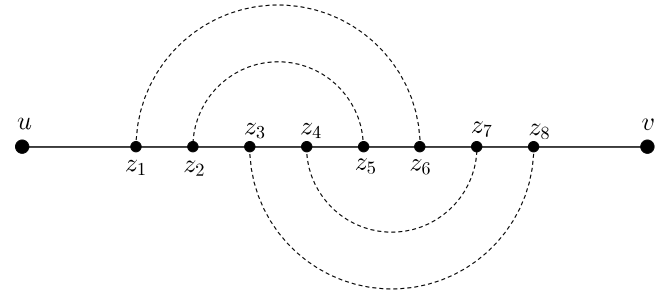

(a) Attaching gadget-subgraphs on $e=(u, v)$.

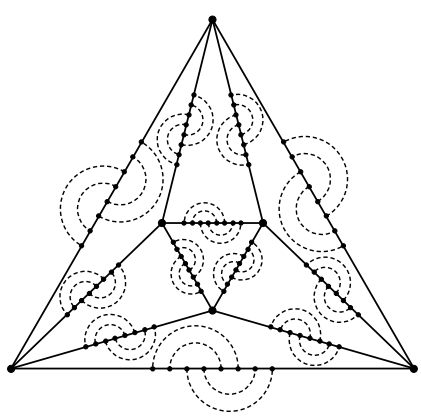

(b) The resulting graph $G_{\text {oct }}^{a u g}$.

Fig. 4. Each dashed edge corresponds to the gadget-subgraph of Fig 5

Initially, we will demonstrate a specific connected 4-regular planar graph that does not admit a realization as a system of circles. This graph will be constructed based on the octahedron graph $G_{o c t}$, augmented by appropriately "attaching" a specific gadget-subgraph to its edges, leading thus to a graph, say $G_{\text {oct }}^{\text {aug }}$, that contains cutvertices and separation pairs (note that any connected 4-regular planar graph is bridgeless [18, pp.34]). The gadget-subgraph, shown in Fig 5a, contains exactly two vertices of degree two, namely $v_{1}$ and $v_{2}$, which are its endpoints. We replace every edge $e=(u, v)$ of $G_{\text {oct }}$ by a path consisting of 8 vertices. Let $u \rightarrow z_{1} \rightarrow \ldots \rightarrow z_{8} \rightarrow v$ be the path replacing $(u, v)$. We associate four copies of the gadget-subgraph having vertices $z_{1}, \ldots, z_{8}$ as their endpoints (see Fig 4a . More precisely, the first gadget-subgraph connects vertices $z_{1}$ and $z_{6}$, the second connects $z_{2}$ and $z_{5}$, the third connects $z_{3}$ and $z_{8}$ and the last connects $z_{4}$ and $z_{7}$. Fig $4 \mathrm{~b}$ depicts the resulting graph $G_{\text {oct }}^{\text {aug }}$. Since the produced graph is planar and each edge $(u, v)$ of $G_{o c t}$ lies on the boundary of two distinct faces, the first two gadget-subgraphs attached at $(u, v)$ lie in one face and the other two lie in the other.

The outerface of the gadget-subgraph consists of vertices $v_{1}, v_{2}, w_{1}, w_{2}$ and $w$ (see Fig [5). If we remove the edges of the outerface, the remaining graph consists of three isolated vertices (namely $v_{1}, v_{2}$ and $w$ ) and two disjoint graphs that are subdivisions of the octahedron graph (refer to the gray-shaded graphs of Fig 5a The properties of the gadget-subgraph, that we will investigate in this section are independent of these two graphs, i.e., any simple planar graph satisfying the following degree condition can be used instead: Every vertex is of degree 4 except for exactly one vertex on the outerface that is of degree 2 (refer to vertices $w_{1}$ and $w_{2}$ of the gray-shaded graphs of Fig $5 \mathrm{a}$. The general situation is shown in Fig $5 \mathrm{~b}$, where the subgraphs are drawn as self-loops at vertices $w_{1}$ and $w_{2}$. For convenience, we will refer to these subgraphs as loop-subgraphs.

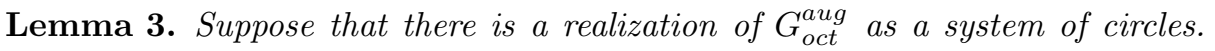
Then, any gadget-subgraph in this realization contributes two tangent circles $C_{1}$ and $C_{2}$, so that circle $C_{i}$ contains vertices $\left\{v_{i}, w_{i}, w\right\}, i=1,2$, and, vertex $w$ is defined by their tangent point. 


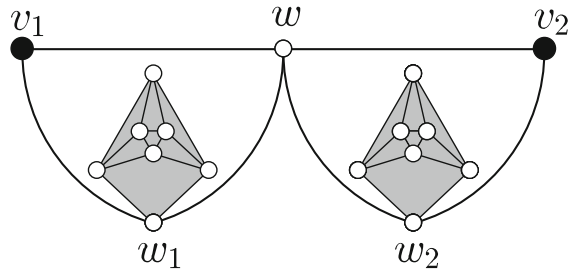

(a) The gadget-subgraph.

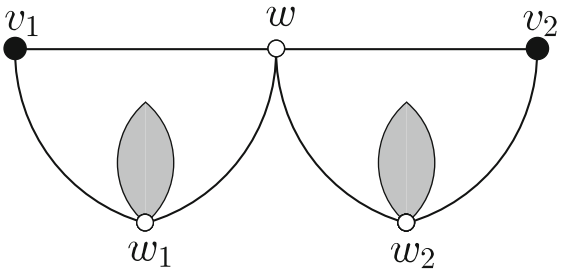

(b) Abstraction of the gadget-subgraph.

Fig. 5. Illustrations of the gadget-subgraph

Proof. Suppose that there is a realization of $G_{\text {oct }}^{\text {aug }}$ as a system of circles and consider a copy of the gadget-subgraph in this realization. Since every vertex is defined by exactly two circles, and $w_{i}$ is a cutvertex, it follows that the edges $\left(v_{i}, w_{i}\right)$ and $\left(w_{i}, w\right)$ belong to the same circle, $i=1,2$. Let $C_{i}$ be the circle that contains $\left(v_{i}, w_{i}\right)$ and $\left(w_{i}, w\right)$ and $C_{i}^{\prime}$ the circle that contains $\left(v_{i}, w\right), i=1,2$. We claim that $C_{i}=C_{i}^{\prime}, i=1,2$. Indeed, suppose that $C_{1} \neq C_{1}^{\prime}$. Since vertex $w$ is defined by exactly two circles, we have that $\left\{C_{1}, C_{1}^{\prime}\right\}=\left\{C_{2}, C_{2}^{\prime}\right\}$, which also implies that $C_{2} \neq C_{2}^{\prime}$. Then, $C_{1}$ and $C_{1}^{\prime}$ have at least three points in common, namely vertices $v_{1}, v_{2}$ and $w$, which suggests that $C_{1}=C_{1}^{\prime}$; a contradiction.

From the above, it follows that each gadget-subgraph has unique realization as a system of circles in the realization of $G_{\text {oct }}^{\text {aug }}$ (ignoring the different realizations of the loop-subgraph as system of circles, and the relative positions of the two touching circles $C_{1}$ and $C_{2}$ ), which is illustrated in Figure 6 .

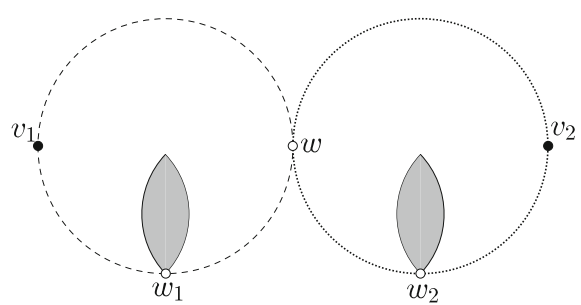

(a)

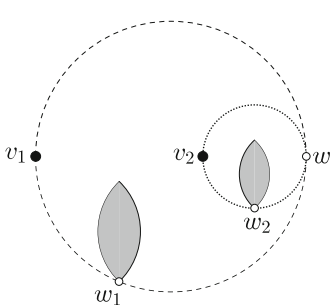

(b)

Fig. 6. Realizations of the gadget-subgraph as system of circles

Lemma 4. Let $e=(u, v)$ be an arbitrary edge of $G_{\text {oct }}$. Augment $G_{\text {oct }}$ by replacing edge $e$ with a path of length two, say $u \rightarrow v_{1} \rightarrow v_{2} \rightarrow v$, and attach a gadget-subgraph, so that $v_{1}$ and $v_{2}$ are its endpoints. Then, in any realization of the produced graph as a system of circles, the realization of the gadget-subgraph and the realization of $G_{\text {oct }}$ are independent, i.e., any circle contains edges that belong exclusively either to the gadget-subgraph or to $G_{\text {oct }}$. 
Proof. Refer to Fig 7a, By Lemma 3, it follows that edges $\left(v_{i}, w_{i}\right),\left(v_{i}, w\right)$ and $\left(w_{i}, w\right)$ belong to the same circle, say $C_{i}, i=1,2$. Then, the edges $\left(u, v_{1}\right),\left(v_{1}, v_{2}\right)$ and $\left(v_{2}, v\right)$ belong to a third circle, say $C$, for which $C \neq C_{i}, i=1,2$. Therefore, if we remove $C_{1}$ and $C_{2}$ and the circles representing the loop-subgraphs of the gadget-subgraph, we obtain a representation of the remaining graph (namely of graph $\left.G_{o c t}\right)$, as a system of circles.

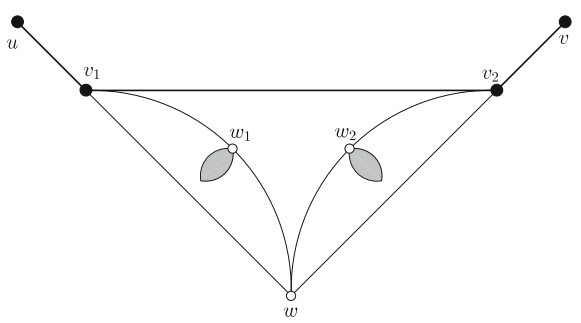

(a)

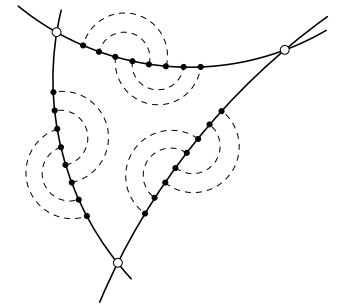

(b)

Fig. 7. (a) Configuration considered in proof of Lemma 4 (b) An embedded triangular face of $G_{\text {oct }}^{\text {aug }}$, in which each dashed edge corresponds to the gadget-subgraph.

Corollary 1. In any realization of $G_{\text {oct }}^{\text {aug }}$ as a system of circles, the realizations of each gadget-subgraph and the realization of $G_{\text {oct }}$ are independent.

Corollary 1 is the key element of our proof. It states that in any realization of $G_{\text {oct }}^{\text {aug }}$ the circles of the gadget-subgraphs are independent of the circles that form $G_{\text {oct }}$, i.e., they only have to touch each other at the endpoints of the gadgetsubgraphs, and do not share any other points. This implies that the drawing of the subgraph where all vertices of the gadget-subgraphs are removed except for their endvertices, will have one of the realizations of $G_{\text {oct }}$ depicted in Figures $1 \mathrm{~b}$ 1d, and the vertices where the gadget-subgraphs are connected to will lie on the corresponding arcs segments implied by these realizations.

The different realizations of the octahedron graph as a system of circles assure that there is always a triangular face that shares no vertex and no edge with the outerface. Hence, there is always a "triangular" face with gadgets that has an embedding similar to that of Fig $7 \mathrm{~b}$ and all gadget-subgraphs attached to its edges cannot admit a realization as the one of Fig $6 \mathrm{~b}$. Note that from the embedding shown in Fig $7 \mathrm{~b}$, one can easily determine all possible such embeddings: For any quadruple of gadget-subgraphs on one edge, we can swap the two pairs of gadget-subgraphs, so that each pair lies in the other face defined by the edge of the octahedron. In the following, we state two useful geometric results.

Lemma 5. Let $C_{1}\left(O_{1}, r_{1}\right)$ and $C_{2}\left(O_{2}, r_{2}\right)$ be two circles, so that $C_{1}$ is tangent to $C_{2}$ at point $p_{1}$ and $C_{2}$ lies entirely in the interior of $C_{1}$. Let $C(O, r)$ be another circle that is tangent to $C_{1}$ at point $p_{2}\left(p_{2} \neq p_{1}\right)$, tangent to $C_{2}$ and lies in the interior of $C_{1}$ (see Fig $8 a$ ). If $\phi$ is the angle $\widehat{p_{1} O_{1} p_{2}}$, then the radius $r$ of $C$ is an increasing function of $\phi, \phi \in(0, \pi]$. 


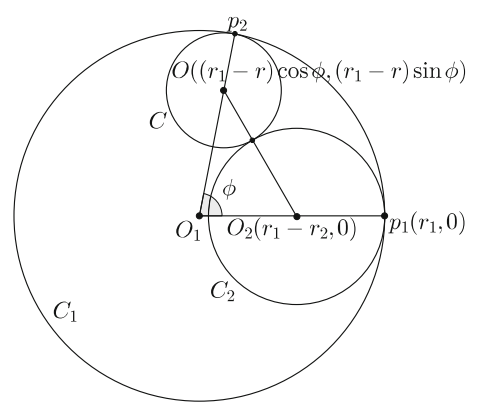

(a)

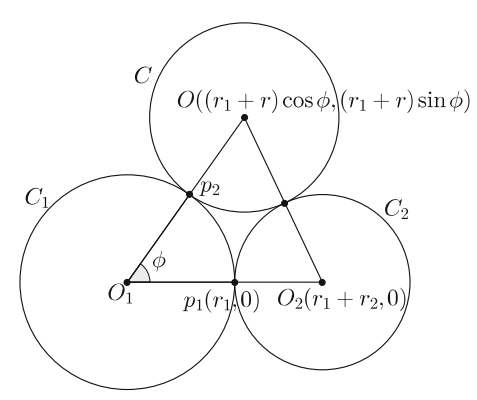

(b)

Fig. 8. (a) Configuration considered in Lemma [5] (b) Configuration considered in Lemma 6 .

Proof. W.l.o.g., we assume that $O_{1}$ coincides with the origin of the cartesian coordinate system and point $p_{1}$ lies on the x-axis, i.e., at point $\left(r_{1}, 0\right)$. Then, the center of circle $C_{2}$ is at point $\left(r_{1}-r_{2}, 0\right)$, while the center of circle $C$ is at point $\left(\left(r_{1}-r\right) \cos \phi,\left(r_{1}-r\right) \sin \phi\right)$, as shown in Fig.8a, Since $C_{2}$ and $C$ are tangent the distance between their centers equals to the sum of their radii, i.e.:

$$
\begin{aligned}
& {\left[\left(r_{1}-r\right) \cos \phi-\left(r_{1}-r_{2}\right)\right]^{2}+\left[\left(r_{1}-r\right) \sin \phi\right]^{2}=\left(r_{2}+r\right)^{2}} \\
& \Rightarrow\left(r_{1}-r_{2}\right)^{2}+\left(r_{1}-r\right)^{2}-2\left(r_{1}-r_{2}\right)\left(r_{1}-r\right) \cos \phi=\left(r_{2}+r\right)^{2} \\
& \Rightarrow r=r_{1}-\frac{2 r_{1} r_{2}}{r_{1}+r_{2}-\left(r_{1}-r_{2}\right) \cos \phi}
\end{aligned}
$$

By the above equation, when $\phi$ is increasing in the interval $(0, \pi], \cos \phi$ is decreasing and $r$ is increasing. Hence, circle $C$ has maximum radius for angle $\phi=\pi$.

Lemma 6. Let $C_{1}\left(O_{1}, r_{1}\right)$ and $C_{2}\left(O_{2}, r_{2}\right)$ be two circles, so that $C_{1}$ is tangent to $C_{2}$ at point $p_{1}$ and $C_{1}$ lies entirely in the exterior of $C_{2}$. Let $C(O, r)$ be another circle that is tangent to $C_{1}$ at point $p_{2}\left(p_{2} \neq p_{1}\right)$, tangent to $C_{2}$ and lies in the exterior of $C_{1}$ (see Fig 86 ). If $\phi$ is the angle $\widehat{p_{1} O_{1} p_{2}}$, then the radius $r$ of $C$ is an increasing function of $\phi, \phi \in\left(0, \arccos \left(\frac{r_{1}-r_{2}}{r_{1}+r_{2}}\right)\right]$.

Proof. The proof of Lemma 6] is similar to the one of Lemma 5. So, we omit the details. We simply mention the corresponding equation for $r$, which is the following:

$$
r=\frac{2 r_{1} r_{2}}{r_{2}-r_{1}+\left(r_{2}+r_{1}\right) \cos \phi}-r_{1} .
$$

Note that circle $C$ does not always exist. For an example, refer to Fig $8 \mathrm{~b}$, when $\phi=\pi / 2$ and $r_{1}>r_{2}$. In particular, for given radii $r_{1}$ and $r_{2}$, angle $\phi$ is bounded from above by value $\arccos \left(\frac{r_{1}-r_{2}}{r_{1}+r_{2}}\right)$, which corresponds to the angle in the extreme case where circle $C$ is of infinite radius and is therefore reduced to the common tangent of circles $C_{1}$ and $C_{2}$.

Lemma 7. Consider a circle $C(O, r)$ and an arc $\widehat{A B}$ of $C$ with $\widehat{A O B}=\phi<\pi$. Let $C_{1}\left(O_{1}, r_{1}\right)$ and $C_{2}\left(O_{2}, r_{2}\right)$ be two tangent circles, that are both tangent to 


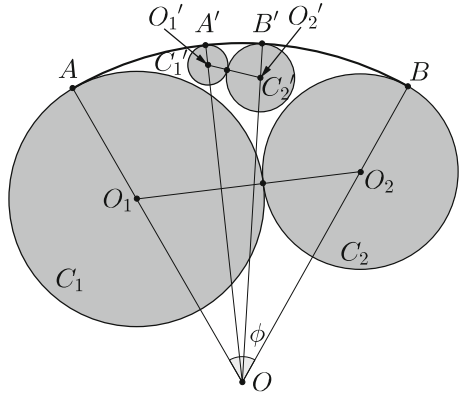

(a)

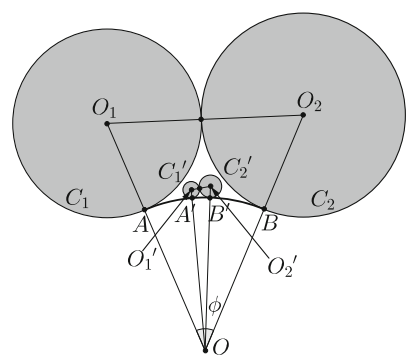

(b)

Fig. 9. Configurations used in Lemma 7

$C$ at points $A$ and $B$ respectively (see Fig 9). Let another such pair of tangent circles $C_{1}^{\prime}\left(O_{1}^{\prime}, r_{1}^{\prime}\right)$ and $C_{2}^{\prime}\left(O_{2}^{\prime}, r_{2}^{\prime}\right)$ that are tangent to $C$ at points $A^{\prime}$ and $B^{\prime}$ respectively (with $A^{\prime}$ and $B^{\prime}$ on the arc between $A$ and $B$ ), so that the two pairs of circles have no crossing and no touching points. Then,

$$
\widehat{A^{\prime} B^{\prime}}<\widehat{A A^{\prime}} \text { and } \widehat{A^{\prime} B^{\prime}}<\widehat{B^{\prime} B}
$$

Proof. By Lemmas 5 and 6, it follows that $r_{i}^{\prime}<r_{j}, i, j \in\{1,2\}$. Consider the circle $C_{1}^{\prime}$. Since $r_{2}^{\prime}<r_{1}$, the previous lemmas assure that $\widehat{A^{\prime} O B^{\prime}}<\widehat{A O A^{\prime}}$. Similarly $\widehat{A^{\prime} O B^{\prime}}<\widehat{B^{\prime} O B}$. So, $A^{\prime} B^{\prime}<\widehat{A A^{\prime}}$ and $\widehat{A^{\prime} B^{\prime}}<\widehat{B^{\prime} B}$, as desired.

Note that Lemma 7 is still true when the four circles lie either in the interior or on the exterior of circle $C$. Let $e=(u, v)$ be an edge of the innermost interior face of the octahedron graph $G_{o c t}$, as shown in Fig $7 \mathrm{~b}$. Assume that in a realization of the octahedron as a system of circles, $e$ is drawn as an arc of a circle $C(O, r)$, with $\widehat{u O v}=\phi<\pi$. The next lemma proves that this assumption leads to a contradiction to the existence of a realization of graph $G_{\text {oct }}^{\text {aug }}$ as a system of circles.

Lemma 8. Consider a circle $C(O, r)$ and assume that edge $e=(u, v) \in E\left[G_{\text {oct }}\right]$ is drawn as an arc segment $\widehat{u v}$ of $C$ such that $\widehat{u O v}=\phi<\pi$. If we attach two pairs of gadget-subgraphs along e, as shown in Fig $4 a$, then the resulting subgraph of $G_{\text {oct }}^{\text {aug }}$ does not admit a realization as a system of circles.

Proof. The existence of only one possible realization of the gadget-subgraphs as system of circles, implies that each gadget-subgraph is drawn as a pair of tangent circles that are also tangent to their endpoints $z_{i}, z_{j}$ along the arc $\overparen{u v}$. By applying Lemma 7 to the pair of gadget-subgraphs with endpoints $z_{1}, z_{6}$ and $z_{2}, z_{5}$, we have:

$$
z_{2} \widetilde{z}_{5}<\widetilde{z}_{1} \widetilde{z}_{2} \text { and } \widetilde{z}_{2} \widetilde{z}_{5}<\widetilde{z}_{5} \widetilde{z}_{6}
$$

Similarly, for the pair of gadget-subgraphs with endpoints $z_{3}, z_{8}$ and $z_{4}, z_{7}$, we have:

$$
\widetilde{z}_{4} z_{7}<\widetilde{z}_{3} \tilde{z}_{4} \text { and } \widetilde{z}_{4} z_{7}<\widetilde{z}_{7} \tilde{z}_{8}
$$


Combining those inequalities and the fact that $\widetilde{z}_{i} z_{j} \leq \overbrace{i^{\prime}} \bar{z}_{j^{\prime}}$ for $i^{\prime} \leq i \leq j \leq j^{\prime}$, we have:

$$
\widetilde{z}_{4} z_{7}<\widetilde{z_{3} z_{4}} \leq \widetilde{z_{2} z_{5}}<\underset{z_{5} z_{6}}{ } \leq \widetilde{z_{4} z_{7}}
$$

that is $\widetilde{z}_{4} z_{7}<\widetilde{z}_{4} \tilde{z}_{7}$, which is a contradiction.

In order to complete the proof that graph $G_{\text {oct }}^{\text {aug }}$ does not admit a realization as a system of circles, it suffices to show that in any realization of $G_{\text {oct }}$, at least one edge of the innermost interior face meets the requirements of Lemma 8 .

Lemma 9. In any realization of the octahedron as a system of circles, at least one edge, say $e=(u, v)$, of the innermost interior face is drawn as an arc of a circle $C(O, r)$ so that $\widehat{u O v}=\phi<\pi$.

Proof. For the first two representations of Fig 1, the result is almost straightforward. More precisely, let $C_{1}\left(O_{1}, r_{1}\right), C_{2}\left(O_{2}, r_{2}\right)$ and $C_{3}\left(O_{3}, r_{3}\right)$ be the circles (white-colored in Fig $1 \mathrm{~b}$ ) that define the innermost interior face (refer to the innermost gray-shaded face of Fig $1 \mathrm{~b}$ ) of the first representation. The three points of this face lie on the edges of the triangle defined by points $O_{1}, O_{2}$ and $O_{3}$, since circles $C_{1}, C_{2}$ and $C_{3}$ are mutually tangent. Then, at least one of the angles of the triangle is less than $\pi$, as desired. In the second representation, the innermost interior face is a circle (refer to the innermost gray-shaded circle of Fig $1 \mathrm{C}$ ) with three distinct points on its boundary. Trivially, at least one of the arcs defined by those points corresponds to an angle that is smaller than $\pi$.

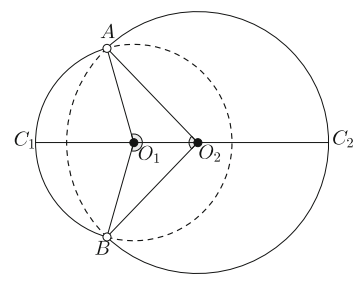

(a)

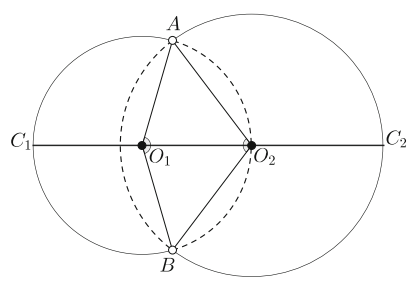

(b)

Fig. 10. Configurations considered in Lemma 9

We now turn our attention to the case where the realization of the octahedron graph as a system of circles is implied by three mutually crossing circles (refer to Fig $1 \mathrm{~d})$. First, consider two intersecting circles $C_{1}\left(O_{1}, r_{1}\right)$ and $C_{2}\left(O_{2}, r_{2}\right)$ at points $A$ and $B$ and assume w.l.o.g. that their centers lie along the x-axis, such that $O_{1}$ is to the left of $O_{2}$ (see Fig 10). We are interested in the angles that correspond to the two arcs of $C_{1}$ and $C_{2}$ that "confine" the common points of the two circles (refer to the dashed drawn arcs incident to $A$ and $B$ in Fig 10). It is not difficult to see that $\widehat{A O_{1} B}$ and $\widehat{A O_{2} B}$ cannot be both greater than $\pi$. Consider now two of the crossing circles of the realization of the octahedron of Fig $1 \mathrm{~d}$, From the above, it follows that at least one of the two arcs that "confine" their common points, corresponds to an angle that is less than $\pi$. 
Since the innermost interior face is also confined by the two arcs, it follows that at least one edge of the innermost interior face has the desired property.

Theorem 2. There exists a simple connected 4-regular planar graph that does not admit a realization as a system of circles.

Proof. Lemma 9 states that in any realization of the octahedron graph as a system of circles, at least one edge of the innermost interior face is drawn as an arc segment of length less than $\pi$. Hence, by Lemma 8 it follows that $G_{\text {oct }}^{\text {aug }}$ does not admit a realization as a system of circles.

Theorem 3. There exists an infinite class of simple connected 4-regular planar graphs that do not admit a realization as a system of circles.

Proof. Recall that in order to obtain $G_{\text {oct }}^{a u g}$, each edge of the octahedron graph was augmented by two pairs of gadget-subgraphs. However, Theorem 2 trivially holds if more than two pairs of gadget-subgraphs are attached to each edge of $G_{\text {oct }}$, defining thus an infinite class of connected 4-regular planar graphs that do not admit a realization as a system of circles. An alternative (and more interesting) class of such graphs can be derived by replacing the octahedron graph of the loop-subgraph of each gadget-subgraph by any 4-regular planar graph, in which one of the edges on its outerface is replaced by a path of length two and the additional vertex implied by this procedure is identified by vertices $w_{1}$ and/or $w_{2}$ of the gadget-subgraph (refer to Fig 5 ).

\section{Conclusion - Open Problems}

In this paper, we proved that every 3-connected 4-regular planar graph admits a realization as a system of touching circles. We also demonstrated that there exist simple 4-regular planar graphs which are not 3-connected and do not admit realizations as system of circles. However, our work raises several open problems.

What is the computational complexity of the corresponding decision problem, i.e., does a given connected 4-regular planar graph admit a realization as a system of circles? Which is the smallest connected 4-regular planar graph not admitting a realization as a system of circles? Is Lovász's conjecture true for biconnected graphs? The octahedron graph admits different realizations as system of circles, in which the number of circles participating in the corresponding realizations also differs. In general, an $n$-vertex 4 -regular planar graph needs at least $(1+$ $\sqrt{1+4 n}) / 2$ and at most $2 n / 3$ circles in order to be realized as a system of circles. What is the minimum number of circles needed in order to realize a given (3connected) 4-regular planar graph as a system of circles? In the context of graph realizations as system of circles, it would be interesting to study the classes of Eulerian and/or $2 k$-regular planar graphs, for $k>2$. Obviously, for these classes of graphs each vertex is defined as the intersection of more than two circles. 


\section{References}

1. Biedl, T., Kant, G.: A Better Heuristic for Orthogonal Graph Drawings. In: van Leeuwen, J. (ed.) ESA 1994. LNCS, vol. 855, pp. 24-35. Springer, Heidelberg (1994)

2. Bollobas, B.: Modern Graph Theory. Springer (1998)

3. Broersma, H., Duijvestijn, A., Göbel, F.: Generating all 3-connected 4-regular planar graphs from the octahedron graph. J. of Graph Theory 17(5), 613-620 (1993)

4. Chernobelskiy, R., Cunningham, K., Goodrich, M., Kobourov, S.G., Trott, L.: Force-Directed Lombardi-Style Graph Drawing. In: van Kreveld, M., Speckmann, B. (eds.) GD 2011. LNCS, vol. 7034, pp. 310-321. Springer, Heidelberg (2012)

5. Duncan, C.A., Eppstein, D., Goodrich, M.T., Kobourov, S.G., Nöllenburg, M.: Drawing Trees with Perfect Angular Resolution and Polynomial Area. In: Brandes, U., Cornelsen, S. (eds.) GD 2010. LNCS, vol. 6502, pp. 183-194. Springer, Heidelberg (2011)

6. Duncan, C.A., Eppstein, D., Goodrich, M.T., Kobourov, S.G., Nöllenburg, M.: Lombardi Drawings of Graphs. J. of Graph Algorithms and Applications 16(1), 85-108 (2011)

7. Erdos, P., Renyi, A., Sos, V.T.: Combinatorial theory and its applications. NorthHolland, Amsterdam (1970)

8. Hlinený, P.: Classes and recognition of curve contact graphs. J. of Combinatorial Theory, Series B 74(1), 87-103 (1998)

9. Hlinený, P., Kratochvíl, J.: Representing graphs by disks and balls (a survey of recognition-complexity results). Discrete Mathematics 229(1-3), 101-124 (2001)

10. Koebe, P.: Kontaktprobleme der konformen Abbildung. Berichte über die Verhandlungen der Sächsischen Akademie der Wissenschaften zu Leipzig, MathematischPhysikalische Klasse 88, 141-164 (1936)

11. Lehel, J.: Generating all 4-regular planar graphs from the graph of the octahedron. J. of Graph Theory 5(4), 423-426 (1981)

12. Leighton, F.T.: New lower bound techniques for VLSI. In: 22nd Ann. IEEE Symp. on Foundations of Computer Science, pp. 1-12. IEEE (1981)

13. Leiserson, C.E.: Area-efficient graph layouts (for VLSI). In: 21st Ann. IEEE Symp. on Foundations of Computer Science, vol. 1547, pp. 270-281. IEEE (1980)

14. Manca, P.: Generating all planar graphs regular of degree four. J. of Graph Theory $3(4), 357-364(1979)$

15. Tamassia, R.: On embedding a graph in the grid with the minimum number of bends. SIAM J. of Computing 16, 421-444 (1987)

16. Tamassia, R., Tollis, I.: Planar grid embedding in linear time. IEEE Transactions on Circuits and Systems (1989)

17. Valiant, L.G.: Universality considerations in VLSI circuits. IEEE Transaction on Computers 30(2), 135-140 (1981)

18. West, D.B.: Introduction to Graph Theory. Prentice Hall (2000) 